\title{
REDUCIBILITIES AMONG DECISION PROBLEMS FOR HNN GROUPS, VECTOR ADDITION SYSTEMS AND SUBSYSTEMS OF PEANO ARITHMETIC
}

\author{
MICHAEL ANSHEL AND KENNETH MCALOON
}

\begin{abstract}
Our purpose is to exhibit reducibilities among decision problems for conjugate powers in HNN groups, reachability sets of vector addition systems and sentences in subsystems of Peano arithmetic, and show that although these problems are not primitive recursively decidable, they do admit decision procedures which are primitive recursive in the Ackermann function.
\end{abstract}

By the class of vector groups VA we understand the HNN groups $G\left(p_{1}, q_{1}, \ldots, p_{k}, q_{k}\right)$ given by

$$
\left(a_{1}, \ldots, a_{k}, b ; a_{1}^{-1} b^{p_{1}} a_{1}=b^{q_{1}}, \ldots, a_{k}^{-1} b^{p_{k}} a_{k}=b^{q_{k}}\right),
$$

where the exponent pairs $p_{i}, q_{i}$ occurring in (I) are positive and relatively prime. (For concepts and results of a group-theoretic nature not explicitly discussed here the reader should consult Lyndon and Schupp [5].)

Let $G$ be a vector group, $m$ a positive conjugate power of $l$ in $G$ when $b^{m}=x b^{l} x^{-1}$, where $x$ in $G$ is given by a positive word in the generators $a_{1}, \ldots, a_{k}, b$ of $G$ (i.e. one which involves no negative exponents). The set of positive conjugate powers of $l$ in $G$, or positive conjugate power set is denoted $\operatorname{PCP}(l, G)$.

By the equality problem for positive conjugate power sets, we mean the question of determining for any integers $l_{1}, l_{2}$ and vector groups $G_{1}, G_{2}$, whether $\operatorname{PCP}\left(l_{1}, G_{1}\right)=$ $\operatorname{PCP}\left(l_{2}, G_{2}\right)$. The special equality problem is to decide the equality problem in those cases where $l_{1}=l_{2}$ and $G_{2}$ arises from $G_{1}$ by removing a particular generating symbol $a_{i}$ and its corresponding defining relation $a_{i}^{-1} b^{p_{i}} a_{i}=b^{q_{i}}$ from the presentation of $G_{1}$ as in (I). The finite special equality problem is to decide the special equality problem in those cases where $\operatorname{PCP}\left(l_{1}, G\right)$ is finite.

We identify a decision problem with the set of Gödel numbers of its instances and use this identification to discuss the complexity of the problem.

A function $g$ is primitive recursive in a function $f$ iff $g$ is in the class obtained by primitive recursion and composition from $f$ together with the usual initial functions.

It is shown in Anshel [1] that the special equality problem for vector groups is undecidable. In contrast, we will prove

THEOREM 1. The finite special equality problem for vector groups is

(i) decidable but not primitive recursive,

(ii) primitive recursive in the Ackermann function.

Received by the editors June 27, 1981.

1980 Mathematics Subject Classification. Primary 20F10, 03D40, 03F30; Secondary 68C99.

(C)1983 American Mathematical Society $0002-9939 / 83 \$ 1.00+\$ .25$ per page 
By a vector addition system (of dimension $n$ ) we mean a pair $(d, W), d$ in $N^{n}, W$ a finite subset of $Z^{n}$ (where $N$ and $Z$ denote the nonnegative integers and integers respectively). Let us call $d^{\prime}$ reachable in $(d, W)$ if $d=d^{\prime}$ or $d^{\prime}=d+w_{1}+\cdots+w_{t}$, $w_{i}$ in $W$ and $d+w_{1}+\cdots+w_{s}$ in $N^{n}, s=1, \ldots, t$.

Let $R(d, W)$ denote the reachable vectors in $(d, W)$ or reachability set of $(d, W)$. Let $K$ be a class of vector addition systems. By the equality problem for $K$ we mean the problem of deciding for arbitrary $\left(d_{1}, W_{1}\right),\left(d_{2}, W_{2}\right)$ in $K$ whether $R\left(d_{1}, W_{1}\right)=$ $R\left(d_{2}, W_{2}\right)$. The special equality problem for $K$ is to decide the equality problem in those cases where $d_{1}=d_{2}$ and $W_{2}$ arises from $W_{1}$ by removal of a vector.

Let VAS denote the class of vector addition systems and BVAS denote the bounded vector addition systems (i.e. $(d, W)$ is in BVAS iff $R(d, W)$ is finite). (For a general introduction to vector addition systems via their graph-theoretic counterparts, Petri nets, the reader should consult Peterson [9].)

We say that a function $f$ is Kalmar-elementary or simply elementary, if $f$ can be obtained by composition, explicit transformation and limited recursion from the exponentiation and successor functions. Thus $f$ is elementary if $f$ is in the Grzegorcyck class $\mathcal{E}_{3}$; in machine theoretic terms $f$ is elementary if $f$ can be computed by a Turing machine bounded in space or time by an iterated exponential function of the input length, that is, predictably computable in the sense of Ritchie. For an exposition of these results the reader may consult Yasuhara [12].

We say that a decision problem $A$ is elementarily reducible to $B$ provided there is an elementary function $g: A \rightarrow B$ such that each instance $x$ of $A$ may be decided by computing the instance $g(x)$ of $B$ and then deciding $g(x)$. We say that $A$ and $B$ are elementarily bireducible whenever each problem is elementarily reducible to the other.

We observe that if $A$ and $B$ are elementarily bireducible, then $A$ is primitive recursively decidable (in the Ackermann function) precisely when $B$ is primitive recursively decidable (in the Ackermann function).

LEMMA 1. The ( finite) special equality problem for vector groups is elementarily bireducible to the special equality problem for (bounded) vector addition systems.

Proof. The following reduction of the (finite) special equality problem for vector groups to the special equality problem for (BVAS) VAS is based on one in [1]. We may assume w.l.o.g. that $l$ is a nonzero integer, $G_{1}$ is given as in (I) and $G_{2}$ arises from $G_{1}$ by removal of $a_{i_{0}}$ and $a_{i_{0}}^{-1} b^{p_{i_{0}}} a_{i_{0}}=b^{q_{i_{0}}}$.

Reduction Algorithm A.

Step 1. Input $l, G_{1}, G_{2}$.

Step 2. Create a list $c_{1}, \ldots, c_{n}$ of the positive prime divisors of the exponents of $G_{1}$.

Step 3. For $i=1, \ldots, k$, create the exponent lists $e_{1 i}, \ldots, e_{n i}$ and $e_{1 i}^{\prime}, \ldots, e_{n i}^{\prime}$ where $p_{i}=c_{1}^{e_{n i}} \cdots c_{n}^{e_{n i}}$ and $q_{i}=c^{e_{1 i}^{\prime}} \cdots c_{n}^{e_{n i}^{\prime}}$.

Step 4. Compute the list $s_{1}, \ldots, s_{n}$ of nonnegative integers uniquely determined by the equation $l=c_{1}^{s_{1}} \cdots c_{n}^{s_{n}} l^{\prime}$ where $l^{\prime}$ is relatively prime to each $c_{i}, i=1, \ldots, n$.

Step 5. Create the vector addition systems $\left(d_{1}, W_{1}\right),\left(d_{2}, W_{2}\right)$ where $d=\left(s_{1}, \ldots, s_{n}\right)$, $W_{1}$ consists of $w_{1}, \ldots, w_{k}$ and $W_{2}=W_{1}-\left\langle w_{i_{0}}\right\rangle$, such that $w_{j}=\left(u_{j 1}, \ldots, u_{j n}\right)$ with 
$u_{j t}, t=1, \ldots, n$, given as in the following manner:

$$
u_{j t}= \begin{cases}e_{j t}, & \text { if } e_{j t} \neq 0 \\ -e_{j t}^{\prime}, & \text { otherwise. }\end{cases}
$$

Step 6. Output $\left(d_{1}, W_{1}\right),\left(d_{2}, W_{2}\right)$.

From Lemma 1 of [1] we have that $\operatorname{PCP}\left(l, G_{1}\right)=\operatorname{PCP}\left(l, G_{2}\right)$ iff $R\left(d, W_{1}\right)=$ $R\left(d, W_{2}\right)$. Moreover, $\operatorname{PCP}\left(l, G_{1}\right)$ is finite iff $R\left(d, W_{1}\right)$ is finite. That algorithm $\mathrm{A}$ above defines an elementary function follows from the fact that the lists created in Steps 2-4 are computable by elementary functions.

The required reduction of the special equality problem for (B)VAS to the (finite) special equality problem for vector groups is exhibited below. Let $\left(d, W_{1}\right),\left(d, W_{2}\right)$ be vector addition systems where $d=\left(s_{1}, \ldots, s_{n}\right), W_{1}=\left\langle w_{1}, \ldots, w_{k}\right\rangle, W_{2}=W_{1}-$ $\left\langle w_{i_{0}}\right\rangle$ and $w_{i}=\left(u_{1 i}, \ldots, u_{n i}\right), i=1, \ldots, k$. Also let $c_{j}$ denote the $j$ th positive prime, $j=1, \ldots, n$.

Reduction Algorithm B.

Step 1. Input $\left(d, W_{1}\right),\left(d, W_{2}\right)$.

Step 2. Compute $l=c_{1}^{s_{1}} \cdots c_{n}^{s_{n}}$.

Step 3. For $i=1, \ldots, k$, compute $p_{i}=c_{1}^{e_{11}} \cdots c_{n}^{e_{i n}}, q_{i}=c_{1}^{e_{1}^{\prime}} \cdots c_{n}^{e_{n i}^{\prime}}$, such that $j=1, \ldots, n$,

$$
e_{i j}=\left\{\begin{array}{ll}
u_{i j}, & \text { if } u_{i j} \geqslant 0, \\
0, & \text { otherwise. }
\end{array} \quad e_{i j}^{\prime}= \begin{cases}-u_{i j}, & \text { if } u_{i} \leqslant 0 \\
0, & \text { otherwise. }\end{cases}\right.
$$

Step 4. Create the vector groups $G_{1}, G_{2} ; G_{1}=G\left(p_{1}, q_{1}, \ldots, p_{k}, q_{k}\right)$ and $G_{2}$ obtained from $G_{1}$ by omitting $a_{i_{0}}$ and the relation $a_{i_{0}}^{-} b^{p_{i_{0}}} a_{i_{0}}=b^{q_{i_{0}}}$ from the presentation of $G_{1}$.

Step 5. Output $l, G_{1}, G_{2}$.

Now $R\left(d, W_{1}\right)=R\left(d, W_{2}\right)$ iff $\operatorname{PCP}\left(l, G_{1}\right)=\operatorname{PCP}\left(l, G_{2}\right)$, and $R\left(d, W_{1}\right)$ is finite iff $\operatorname{PCP}\left(l, G_{1}\right)$ is finite. That algorithm $\mathrm{B}$ defines an elementary function follows from the fact that $l, p_{i}, q_{i}, i=1, \ldots, k$, are computable by elementary functions.

The boundedness problem for a class $K$ of vector addition systems (resp. vector groups) is to decide for any $(d, W)$ in $K$ (resp. $G$ in $K$ and integer $l$ ) whether $R(d, W)$ is finite (resp. $\operatorname{PCP}(l, G)$ is finite).

COROLlaRY 1. The boundedness problem for vector groups is elementary recursive.

PROOF. Rackoff [10] has shown that the boundedness problem for VAS is elementary recursive. The result is then immediate from Lemma 1 above.

LEMMA 2. The special equality problem for bounded vector addition systems is (i) decidable, (ii) not primitive recursive but (iii) primitive recursive in the Ackermann function.

Proof. (i) With each $(d, W)$ in VAS we can recursively associate its Karp-Miller coverability tree $T$ which is finite (cf. Karp and Miller [3]). Moreover, if $R(d, W)$ is finite, then the nodes of $T$ consist precisely of the reachable vector of $(d, W)$. Thus 
given $\left(d_{1}, W_{1}\right)$ and $\left(d_{2}, W_{2}\right)$ in BVAS, to compare their reachability sets, it is enough to compare their associated Karp-Miller coverability trees.

(ii) It follows from the proof of the reduction of the reachability set inclusion problem (which asks if $R\left(d_{1}, W_{1}\right)$ is contained in $R\left(d_{2}, W_{2}\right)$ ) to the equality problem, as given in Hack [2], that the inclusion problem for (B)VAS is elementarily bireducible to the special equality problem for (B)VAS (a fact also understood by Mayr [6]). It is a consequence of the Corollary to Theorem 7 of Mayr [6] that the inclusion problem for BVAS is not primitive recursively solvable, and neither is the special equality problem for BVAS.

(iii) By (i) it is enough to give an upper bound for the map $K M:(d, W) \mapsto T$ which associates with each $(d, W)$ in VAS its Karp-Miller coverability tree $T$. The proof that $K M$ is primitive recursive in the Ackermann function is carried out in McAloon [7]. The main steps are the following: Let $I \Sigma$, be the subsystem of Peano Arithmetic obtained by restricting the induction scheme to $\Sigma_{1}^{0}$-formulas (i.e. to those of the form $(\exists \vec{x}) \Phi(\vec{x}, \vec{n})$ where $\Phi$ contains only bounded quantifiers). Now given $(d, W)$, one has

$$
I \Sigma_{1}+\exists T(T=K M(\bar{d}, \bar{w})) .
$$

To obtain $(*)$ one has to analyze the proof that the tree $T$ associated with $(d, W)$ is, in fact, finite. One then has

$$
I \Sigma_{1}+1-\text { Consistency }\left(I \Sigma_{1}\right) \vdash K M \text { is a total function }
$$

(where 1 - Consistency $\left(I \Sigma_{1}\right)$ asserts that every $\Sigma_{1}^{0}$-sentence provable in $I \Sigma_{1}$ is true). By applying results of Kirby and Paris [4] and Paris [8], all recursive functions provably total in $I \Sigma_{1}+1-$ Consistency $\left(I \Sigma_{1}\right)$ are primitive recursive in the Ackermann function.

Observe that Theorem 1 follows directly from Lemmas 1 and 2 above.

Let (B) $\mathrm{VAS}_{n}$ denote the class of (bounded) vector addition systems of dimension $n$. For each of the decision problems discussed above, there is naturally a version at dimension $n$. We have the following result.

THEOREM 2. Let $n \geqslant 0$; the inclusion, equality and special equality problem for $B V A S_{n}$ are all primitive recursive.

IDEA OF PROOF. For each dimension $n$, we have again by an analysis of the proof that the Karp-Miller coverability tree associated with a system is finite

$$
I \Sigma_{1} \vdash \forall(d, W)\left[\left(d_{1} W\right) \text { of dimension } n \rightarrow \exists T(T=K M(\bar{d}, \bar{W}))\right] .
$$

Hence, by the methods of Proof Theory (cf. Takeuti [11]) or by the methods of Model Theory (cf. Kirby and Paris [4], Paris [8]) the function $K M$ restricted to systems of dimension $n$ is primitive recursive.

REMARK. The above result shows that the complexity of the special equality problem for BVAS comes from the fact that the arbitrary dimensions are considered.

REMARK. The above result also leads to some open questions as to what reducibilities exist among the various decision problems at dimension $n$. From the work of 
Hack [2] we do know that the inclusion problem for (B)VAS at dimension $n$ is polynomial time reducible to the special equality problem for (B)VAS at dimension $n+5$.

Our reductions imply that there should be a notion of dimension of vector groups. The one which emerges is the following: define the dimension of a vector group $G\left(p_{1}, q_{1}, \ldots, p_{k}, q_{k}\right)$ as the number of distinct positive prime divisors of the exponents $p_{i}, q_{i}, i=1_{i}, \ldots, k$. We then have

COROLlaRY 2. Let $n \geqslant 0$; the finite special equality problem for $V A_{n}$, the class of vector groups of dimension $n$, admits a primitive recursive decision procedure.

\section{REFERENCES}

1. M. Anshel, Vector groups and the equality problem for vector addition systems, Math. Comp. 32 (1978), 614-616.

2. M. Hack, The equality problem for vector addition systems is undecidable, Theoret. Comput. Sci. 2 (1976), 77-96.

3. R. M. Karp and R. E. Miller, Parellel program schemata, J. Comput. System Sci. 3.(1969), 147- 195.

4. L. Kirby and J. Paris, Initial segments of models of arithmetic, Set Theory and Hierarchy Theory V. Lecture Notes in Math., Vol. 619, Springer-Verlag, Berlin, 1977.

5. R. C. Lyndon and P. E. Schupp, Combinatorial group theory, Springer-Verlag, Berlin, 1977.

6. E. Mayr, The complexity of the finite containment problem for Petri nets, Laboratory for Computer Science, Technical Report No. 181, M.I.T., Cambridge, Mass., 1977.

7. K. McAloon, Petri nets and large finite sets, Theoret. Comput. Sci. (to appear).

8. J. Paris, Hierarchy of cuts in models of arithmetic, Model Theory of Algebra and Arithmetic, Lecture Notes in Math., Vol. 834, Springer-Verlag, Berlin, 1980.

9. J. L. Peterson, Petri nets and the modelling of systems, Prentice-Hall, Englewood Cliffs, N. J., 1981.

10. C. Rackoff, The covering and houndedness problems for vector addition systems, Theoret. Comput. Sci. 6 (1978), 223-231.

11. G. Takeuti, Proof theory, North-Holland, Amsterdam, 1975.

12. A. Yashuhara, Recursive function theory and logic, Academic Press, New York, 1971.

Department of Computer Sciences, City College, City University of New York, New York, NEW YORK 10031

Department of Computer and Information Science, Brooklyn College, City University of NEW YORK, BROOKLYN, NEW YORK 11210 